\title{
Solving a Mixed Problem with Almost Regular Boundary Condition By the Contour Integral Method
}

\author{
S. T. Aleskerova ${ }^{1}$
}

${ }^{1}$ Institute of Mathematics and Mechanics of ANAS, Baku, Azerbaijan

Correspondence: S. T. Aleskerova, Azerbaijan State Pedagogical University, Azerbaijan, Department of Mathematical Analysis. E-mail: sabina.alesgerova75@gmail.com

Received: October 21, 2016 Accepted: December 5, 2016 Online Published: January 23, 2016

doi:10.5539/jmr.v9n1p158 URL: http://dx.doi.org/10.5539/jmr.v9n1p158

\begin{abstract}
In the paper, a mixed problem for $\lambda$-complex parameter dependent, fourth order partial equation is considered. The estimations for the Green function for the eigenvalues of the considered problem outside of $\delta$ were obtained within the regular boundary condition. The solution of the mixed problem was given in the contour integral form.
\end{abstract}

Keywords: fundamental solutions, asymptotic, mixed problem, boundary conditions, sector, contour, eigenvalues, spectral problem

\section{Introduction}

The considered equation arises when considering heat and diffusion processes the boundary conditions are given in the general form. The paper deals with finding the solution of the mixed problem within second order almost regular boundary conditions. The solution of the problem is found by M.L. Rasulov's contour integral method. The studied mixed problem, mathematically given as follows. The following problem is considered

$$
\begin{aligned}
& \frac{\partial^{2} u(x, t)}{\partial t^{2}}-\left(p_{1}+p_{2^{\circ}}\right) \frac{\partial^{3} u(x, t)}{\partial t \partial x^{2}}+p_{1} p_{2} \frac{\partial^{4} u(x, t)}{\partial x^{4}}= \\
& =a(x) \frac{\partial u(x, t)}{\partial t}+b(x) \frac{\partial^{2} u(x, t)}{\partial x^{2}}, 0<x<1, t>0 \\
& L_{1} \equiv \sum_{k=1}^{4} \alpha_{1 k} \frac{\partial^{(k-1)} u(0, t)}{\partial x^{k-1}}+\sum_{k=1}^{4} \beta_{1 k} \frac{\partial^{(k-1)} u(1, t)}{\partial x^{k-1}}=0 \\
& L_{2} \equiv \sum_{k=1}^{4} \alpha_{2 k} \frac{\partial^{(k-1)} u(0, t)}{\partial x^{k-1}}+\sum_{k=1}^{4} \beta_{2 k} \frac{\partial^{(k-1)} u(1, t)}{\partial x^{k-1}}=0 \\
& L_{3} \equiv \sum_{k=1}^{4} \alpha_{3 k} \frac{\partial^{(k-1)} u(0, t)}{\partial x^{k-1}}+\sum_{k=1}^{4} \beta_{3 k} \frac{\partial^{(k-1)} u(1, t)}{\partial x^{k-1}}=0 \\
& L_{4} \equiv \sum_{k=1}^{4} \alpha_{4 k} \frac{\partial^{(k-1)} u(0, t)}{\partial x^{k-1}}+\sum_{k=1}^{4} \beta_{4 k} \frac{\partial^{(k-1)} u(1, t)}{\partial x^{k-1}}=0 \\
& u(x, 0)=\varphi(x)
\end{aligned}
$$

where $a(x), b(x), \varphi(x)$ are complex valued functions. $p_{1}, p_{2}, \alpha_{i j}, \beta_{i j}(i, j=\overline{1,4})$ are complex numbers. The conditions $\operatorname{Re} p_{1}>0$ and $\operatorname{Re} p_{2}>0$ are satisfied.

The following spectral problem corresponding to mixed problem ??-?? is constructed 


$$
\begin{gathered}
p_{1} p_{2} y^{I V}-\left(p_{1}+p_{2}\right) \lambda^{2} y^{\prime \prime}+\lambda^{4} y-b(x) y^{\prime \prime}-a(x) \lambda^{2} y=f(x, \lambda) \\
L_{k}(y)=0
\end{gathered}
$$

where

$$
f(x, \lambda)=\lambda^{2} \varphi(x)+\psi(x)-a(x) \varphi(x)-\left(p_{1}+p_{2}\right) \varphi^{\prime \prime}(x) .
$$

Partition the $\lambda$-complex plane into eight sectors

$$
\begin{aligned}
& S_{1}=\left\{\lambda \mid \lambda_{2}>k_{1} \lambda_{1} ; \lambda_{2}<k_{4} \lambda_{1}\right\}, \\
& S_{2}=\left\{\lambda \mid \lambda_{2}>k_{4} \lambda_{1} ; \lambda_{2}<k_{3} \lambda_{1}\right\}, \\
& S_{3}=\left\{\lambda \mid \lambda_{2}>k_{3} \lambda_{1} ; \lambda_{2}<k_{2} \lambda_{1}\right\}, \\
& S_{5}=\left\{\lambda \mid \lambda_{2}>k_{1} \lambda_{1} ; \lambda_{2}<k_{4} \lambda_{1}\right\}, \\
& S_{6}=\left\{\lambda \mid \lambda_{2}>k_{4} \lambda_{1} ; \lambda_{2}<k_{3} \lambda_{1}\right\}, \\
& S_{7}=\left\{\lambda \mid \lambda_{2}>k_{3} \lambda_{1} ; \lambda_{2}<k_{2} \lambda_{1}\right\}, \\
& S_{8}=\left\{\lambda \mid \lambda_{2}>k_{1} \lambda_{1} ; \lambda_{2}<k_{2} \lambda_{1}\right\},
\end{aligned}
$$

where

$$
\begin{gathered}
k_{1}=\operatorname{ctg} \psi_{1} ; k_{2}=\frac{\left|\omega_{3}\right| \cos \psi_{3}-\left|\omega_{1}\right| \cos \psi_{1}}{\left|\omega_{3}\right| \sin \psi_{3}-\left|\omega_{1}\right| \sin \psi_{1}} ; \\
k_{3}=\operatorname{ctg} \psi_{3} ; k_{4}=\frac{\left|\omega_{1}\right| \cos \psi_{1}+\left|\omega_{3}\right| \cos \psi_{3}}{\left|\omega_{1}\right| \sin \psi_{1}+\left|\omega_{3}\right| \sin \psi_{3}} ; \\
\omega_{1}=\left|\omega_{1}\right| e^{\psi_{1} i} ; \omega_{2}=-\omega_{1} ; \omega_{3}=\left|\omega_{3}\right| e^{\psi_{3} i} ; \omega_{4}=-\omega_{3} ; \\
\left|\omega_{1}\right|=\left|p_{1}\right|^{-\frac{1}{2}} ;\left|\omega_{3}\right|=\left|p_{3}\right|^{-\frac{1}{2}} ; \\
\psi_{k}=-\frac{1}{2} \operatorname{arctg} \frac{\operatorname{Im} P_{k}}{\operatorname{Re} P_{k}}, k=1,3, \\
0<\psi_{3}<\psi_{1}<\frac{\pi}{4} ;\left|\omega_{3}\right| \sin \psi_{3}-\left|\omega_{1}\right| \sin \psi_{1}>0 .
\end{gathered}
$$

In each sector of $S p(p=\overline{1,8})$ we find the asymptotics of fundamental solutions of equation 4 in the following way

$$
\frac{d^{k} y_{m}(x, \lambda)}{d x^{k}}=\left(\lambda \omega_{m}\right)^{k}\left[1+\frac{1}{\lambda} y_{m k}^{1}(x)+\frac{1}{\lambda^{2}} y_{m k}^{2}(x)+\frac{E_{m k}(x, \lambda)}{\lambda^{3}}\right] e^{\lambda \omega_{m} x}
$$

where 


$$
\begin{gathered}
y_{m k}^{1}(x)=\frac{1}{4 q \omega_{m}^{3}+2 p \omega_{m}}\left[\int_{0}^{x} a(\xi) d \xi+\omega_{m}^{2} \int_{0}^{x} b(\xi) d \xi\right] \\
y_{m k}^{2}(x)=\frac{1}{4 q \omega_{m}^{3}+2 p \omega_{m}}\left[\int_{0}^{x}\left(a(\xi) d \xi+\omega_{m}^{2} b(\xi)\right) y^{1}(\xi) d \xi\right]- \\
-\frac{6 q \omega_{m}^{2}+p}{4 q \omega_{m}^{3}+2 p \omega_{m}} \frac{d y_{m k}^{1}(x)}{d x}, \\
q=p_{1} p_{2}, p=-\left(p_{1}+p_{2}\right)
\end{gathered}
$$

The functions $E_{m k}(x, \lambda)(m=\overline{1,4} ; k=\overline{0,3})$ are analytic functions and for large values of $|\lambda|$ they are bounded functions. Let us give the following theorem connected with the asymptotics of eigen numbers of spectral problem (4)-(5) and the Green function.

Theorem 1. Assume that the coefficients of equation (4) and boundary conditions (5) satisfy the following conditions.

$$
\begin{gathered}
\operatorname{Re} p_{1}>0, \operatorname{Re} p_{2}>0, a(x), b(x) \in C^{1}[0,1], \\
L\left(\alpha_{3}, \alpha_{4}, \beta_{3}, \beta_{4}\right)=0, L\left(\alpha_{3}, \alpha_{4}, \beta_{2}, \beta_{4}\right)=0, L\left(\alpha_{2}, \alpha_{4}, \beta_{3}, \beta_{4}\right)=0 \\
L\left(\alpha_{2}, \alpha_{3}, \alpha_{4}, \beta_{4}\right) \neq 0, L\left(\alpha_{4}, \beta_{2}, \beta_{3}, \beta_{4}\right) \neq 0 .
\end{gathered}
$$

Then the Green function of eigen-numbers of the spectral problem out of the vicinity of $\delta$ has the following estimation

$$
|G(x, \xi, \lambda)| \leq \frac{M}{|\lambda|^{2}}, \lambda \in S_{p}(p=\overline{1,8}),|\lambda \rightarrow+\infty| .
$$

Thus the following formula is valid for the asymptotics of the eigen-numbers of spectral problem (4), (5)

$$
\begin{gathered}
\lambda_{k v}=-\frac{1}{\omega_{k}}\left\{\ln \left|\frac{2 \pi v A_{k}}{\omega_{k}}\right|+i\left[2 \pi v+\frac{\pi}{2}(2-\operatorname{sgn} v)+\arg A_{k}\right]\right\}+ \\
+O\left(\frac{\ln |v|}{v}\right), k=1,2,3,4 ;(-1)^{k} v \rightarrow+\infty,
\end{gathered}
$$

where $A_{k}$ are complex numbers dependent on the coefficients of equation (4) and boundary conditions (5)

$$
L\left(\gamma_{n}^{1}, \gamma_{m}^{2}, \gamma_{p}^{3}, \gamma_{q}^{4}\right)=\left|\begin{array}{llll}
\gamma_{1 n}^{1} & \gamma_{1 m}^{2} & \gamma_{1 p}^{3} & \gamma_{1 p}^{4} \\
\gamma_{2 n}^{1} & \gamma_{2 m}^{2} & \gamma_{2 p}^{3} & \gamma_{2 p}^{4} \\
\gamma_{3 n}^{1} & \gamma_{3 m}^{2} & \gamma_{3 p}^{3} & \gamma_{3 p}^{4} \\
\gamma_{4 n}^{1} & \gamma_{4 m}^{2} & \gamma_{4 p}^{3} & \gamma_{4 p}^{4}
\end{array}\right|
$$

In the paper the following theorem is proved.

Theorem 2. The coefficients of equation (1), boundary conditions (2) and initial conditions (3) satisfy the following conditions

$$
\begin{gathered}
\operatorname{Re} p_{1}>0, \operatorname{Re} p_{2}>0, a(x), b(x) \in C^{2}[0,1], \\
\varphi(x) \in C^{3}[0,1], \psi(x) \in C^{2}[0,1],
\end{gathered}
$$




$$
\begin{gathered}
\varphi(0)=\varphi(1)=\varphi^{\prime}(0)=\varphi^{\prime}(1)=\varphi^{\prime \prime}(0)=\varphi^{\prime \prime}(1)=0, \\
\psi(0)=\psi(1)=\psi^{\prime}(0)=\psi^{\prime}(1)=0, \\
L\left(\alpha_{3}, \alpha_{4}, \beta_{3}, \beta_{4}\right)=L\left(\alpha_{3}, \alpha_{4}, \beta_{2}, \beta_{4}\right)=L\left(\alpha_{2}, \alpha_{4}, \beta_{3}, \beta_{4}\right)=0 \\
L\left(\alpha_{2}, \alpha_{3}, \alpha_{4}, \beta_{4}\right) \neq 0, L\left(\alpha_{4}, \beta_{2}, \beta_{3}, \beta_{4}\right) \neq 0 .
\end{gathered}
$$

then the solution of problem (1)-(3) will be found in the following contour integral form

$$
U(x, t)=\frac{1}{\pi i} \int_{L_{1}} \lambda y(x, \lambda) e^{\lambda^{2} t} d \lambda,
$$

where the function $y(x, \lambda)$ is the solution of spectral problem (4)-(5).

$L_{1}$ - contour is an infinitely extended unbounded curve and is given by the following formula

$$
\begin{gathered}
L_{1}=\left\{\lambda: \lambda=r e^{i \varphi},|\varphi| \leq \frac{\pi}{4}+\delta, \lambda=R e^{ \pm i\left(\frac{\pi}{4}+\delta\right)}, R \geq r\right\} \\
r>0, \delta=\min \left\{\frac{\pi}{8}-\frac{\psi_{1}}{2}, \frac{\pi}{8}-\frac{\psi_{12}}{2}\right\} .
\end{gathered}
$$

\section{Proof}

$$
\left|e^{\lambda^{2} t}\right|=e^{t R e \lambda^{2}}=e^{t|\lambda|^{2} \cos 2\left(\frac{\pi}{4}+\delta\right)}=e^{-t|\lambda|^{2} \sin 2 \delta}
$$

As $0<\psi_{k}<\frac{\pi}{4}, k=1,2$ then $\sin 2 \delta>0$.

This means that the function $e^{\lambda^{2} t}$ as $t>0, \lambda \in L_{1},|\lambda| \rightarrow+\infty$ decreases exponentially, i.e. the following integrals are regularly convergent

$$
\int_{L_{1}} \lambda \frac{\partial^{p+q}}{\partial t^{p} \partial x^{q}}\left(y(x, \lambda) e^{\lambda^{2} t}\right) d \lambda, p+q \in\{0,1,2,3,4\} .
$$

Show that integral (7) satisfies equation (1)

$$
\begin{gathered}
\frac{\partial^{2} u(x, t)}{\partial t^{2}}-\left(p_{1}+p_{2}\right) \frac{\partial^{3} u(x, t)}{\partial t \partial x^{2}}+p_{1} p_{2} \frac{\partial^{4} u(x, t)}{\partial x^{4}}- \\
-a(x) \frac{\partial u(x, t)}{\partial t}-b(x) \frac{\partial^{2} u(x, t)}{\partial x^{2}}=\frac{1}{\pi i} \int_{L_{1}}\left[\lambda^{4} y(x, \lambda)-\right. \\
\left.-\left(p_{1}+p_{2}\right) \lambda^{2} y^{\prime \prime}(x, \lambda)+p_{1} p_{2} y^{I V}(x, \lambda)-\lambda^{2} a(x) y(x, \lambda)-b(x) y^{\prime \prime}(x, \lambda)\right] \\
\lambda e^{\lambda^{2} t} d \lambda=\frac{1}{\pi i}\left[\varphi(x) \int_{L_{1}} \lambda^{3} e^{\lambda^{2} t} d \lambda+(\psi(x)-a(x) \varphi(x)-\right. \\
\left.\left.-\left(p_{1}+p_{2}\right) \varphi^{\prime \prime}(x)\right) \int_{L_{1}} \lambda e^{\lambda^{2} t} d \lambda\right]=0 .
\end{gathered}
$$

It is easy to show that the following expansion formula is true within the theorem conditions

$$
\sum_{k=1}^{4} \sum_{n=1}^{\infty} \underset{\lambda=\lambda_{k n}}{\operatorname{res}} \lambda^{s+2} \int_{0}^{1} \varphi(\xi) G(x, \xi, \lambda) d \xi=\left\{\begin{array}{cc}
\varphi(x), & s=1 \\
0, & s<1
\end{array} .\right.
$$


Using this formula, we can show that initial conditions (3) are satisfied. As the function $y(x, y)$ is the solution of spectral problem (4), (5) it is easy to verify that integral (7) satisfies boundary conditions (2). The theorem is proved.

\section{Conclusion}

The considered equation is higher order parabolic equation in the sense of Petrovsky. Boundary conditions (2) are called general form boundary conditions. At first a spectral problem corresponding to mixed problem (1)-(3) is constructed. For constructing asymptotics of fundamental solutions of the spectral problem. The $\lambda$-complex plane is divided into 8 sector. The asymptotics of fundamental solutions are found more exactly in each of these sectors. For the first order almost regular case, estimations for the Green function are found far from the $\delta$ vicinity of eigen numbers. Imposing smoothmen conditions and algebraic conditions on initial data, it was possible to find the solution of the problem in the form of the contour integral.

\section{References}

Alesgerova, S. T., Akhmedov, S. Z., \& Abbasova, A. K. (2015). Finding the asymptotics of eigen value of a fourth orde differential operator. Aktualniye problemy gumanitarnikh i yestestvennikh nauk. Journal of Scientific publications, 13-18, Moscow.

Alesgerova, S. T. (2015). Finding the asymptotics of eigen numbers of fourth order differential operator within almost regular condition, News of Pedagogical University, Section of Natural Sciences, 2, 3-8.

Alesgerova, S. T., \& Akhmedov, S. Z. (2015). Finding the asymptotics of eigen numbers for fourth order equation corresponding to a spectral problem, Proceeding of the scientific conference devoted to 85 years of Y.S. Mammadov, BSU, 57-60.

Akhmedov, S. Z., \& Aleskerova, S. T. (2014). On zeros of the characteristical determinant of a spectral problem, dependent on $\lambda$-complex parameter, BDU-nun "Xeberleri", fiz. riy. el. ser., 4, 36-45.

Akhmedov, S. Z., \& Alesgerova, S. T. (2012). Construction of asymptotics of fundamental solutions of equation, BDUnun "Xeberleri", fiz. riy. el. ser., 1, 70-77.

Federyuk, M. V. (1983).Asymptotic methods for linear ordinary differential equations, Moscow, Nauka, Russian, 352.

Mamedov, Yu. A., \& Nagiyeva, R. I. (2010). Mathematical analysis of critical problems of electrodynamics, Baku, Elm, Russian, 181.

Mamedov, Yu. A., \& Akhmedov, S. Z. (2005). Investigation of a characteristical determinant connected with the solution of spectral problem, Vestnik BGU, ser. fiz. math. nauk, Russian, 2, 5-12.

Mammadov, Y. A., \& Alesgerova, T. A. (2012). Estimation of Green function of a spectral problem with quasi-regular boundary condition, Transactions Issue Mathematics, Series of physical-technical and mathematics science, Azerbaijan National Academy of Science, 113-119.

Rasulov, M. L. (1964). Contour integral method, Moscow, Nauka, Russian, 458.

\section{Copyrights}

Copyright for this article is retained by the author(s), with first publication rights granted to the journal.

This is an open-access article distributed under the terms and conditions of the Creative Commons Attribution license (http://creativecommons.org/licenses/by/4.0/). 